\title{
NGC 300 X-1 and IC 10 X-1: a new breed of black hole binary?
}

\author{
R. Barnard, J. S. Clark, and U. C. Kolb
}

The Department of Physics and Astronomy, The Open University, Walton Hall, Milton Keynes MK7 6BT, UK

e-mail: r.barnard@open.ac.uk

Received 30 May 2007 / Accepted 5 July 2008

\section{ABSTRACT}

Context. IC $10 \mathrm{X}-1$ has recently been confirmed as a black hole (BH) + Wolf-Rayet (WR) X-ray binary, and NGC $300 \mathrm{X}-1$ is thought to be. The only other known BH+WR candidate is Cygnus X-3. IC 10 X-1 and NGC 300 X-1 have similar X-ray properties, with $0.3-10 \mathrm{keV}$ luminosities $\sim 10^{38} \mathrm{erg} \mathrm{s}^{-1}$, and their X-ray lightcurves exhibit orbital periods $\sim 30 \mathrm{~h}$.

Aims. We investigate similarities between IC $10 \mathrm{X}-1$ and NGC $300 \mathrm{X}-1$, as well as differences between these systems and the known Galactic BH binary systems.

Methods. We have examined all four XMM-Newton observations of NGC $300 \mathrm{X}-1$, as well as the single XMM-Newton observation of IC $10 \mathrm{X}-1$. For each observation, we extracted lightcurves and spectra from the pn, MOS1 and MOS2 cameras; power density spectra were constructed from the lightcurves, and the X-ray emission spectra were modeled.

Results. Each source exhibits power density spectra that are well described by a power law with index, $\gamma, \sim 1$. Such variability is characteristic of turbulence in wind accretion or disc-accreting X-ray binaries (XBs) in the high state. In this state, Galactic XBs with known BH primaries have soft, thermal emission; however the emission spectra of NGC $300 \mathrm{X}-1$ and IC $10 \mathrm{X}-1$ in the XMM-Newton observations are predominantly non-thermal. Furthermore, the Observation 1 spectrum of NGC 300 X-1 is strikingly similar to that of IC $10 \mathrm{X}-1$.

Conclusions. The remarkable similarity between the behaviour of NGC $300 \mathrm{X}-1$ in Observation 1 and that of IC $10 \mathrm{X}-1$ lends strong evidence for NGC $300 \mathrm{X}-1$ being a BH+WR binary. Our spectral modeling rules out Bondi-Hoyle accretion onto a neutron star (NS) for NGC 300 X-1, but not a disc-accreting NS+WR system, nor a NS low mass X-ray binary (LMXB) that is merely coincident with the WR. We favour disc accretion for both systems, but cannot exclude Bondi-Hoyle accretion onto a BH.

The unusual spectra of NGC 300 X-1 and IC 10 X-1 may be due to these systems existing in a persistently high state, whereas all known BH LMXBs are transient. The violent transition from low state to high state may temporarily eject the disc corona from the $\mathrm{BH}$ LMXBs, drastically reducing the non-thermal component. However, BH XBs in a persistent high state could retain their corona, and hence exhibit a large non-thermal component. LMC X-1 is a BH XB that has only been observed in the high state, and its spectrum is remarkably similar to those of NGC $300 \mathrm{X}-1$ in Observation 1 and IC $10 \mathrm{X}-1$. We therefore classify NGC $300 \mathrm{X}-1$, IC $10 \mathrm{X}-1$ and perhaps LMC X-1 as a new breed of BH XB, defined by their persistently high accretion rates and consequent stable disc configuration and corona. This scenario may also explain the lack of ultraluminous X-ray sources in the canonical soft state.

Key words. X-rays: binaries - X-rays: general - galaxies: individual: NGC 300 - galaxies: individual: IC 10

\section{Introduction}

Characterised by high temperatures and large mass loss rates, Wolf Rayet (WR) stars represent the final stage of stellar evolution prior to supernova for massive stars $\left(>25 M_{\odot}\right.$; Crowther 2007). Their spectra are characterised by strong, broad emission lines, which reveal their chemically evolved nature due to, successively, the CNO (WN stars) and triple- $\alpha$ (WC stars) processes. These products of nuclear burning become visible as the hydrogen rich mantle of the $\mathrm{O}$ star progenitor is stripped away. For single stars this is accomplished via the stellar wind of the progenitor; however binary interaction may also contribute to this process. Indeed, if a stellar merger may be avoided, interaction between the two components of a high mass X-ray binary (OB star and compact object) is thought to lead to a short period WR+compact object binary (WR+co).

Empirically, such systems are exceedingly rare; to date we know of only three possible WR+co systems: Cygnus X-3 in our Galaxy (Lommen et al. 2005), IC 10 X-1 (Clark \& Crowther 2004; Bauer \& Brandt 2004), and most recently, NGC 300 X-1 (Carpano et al. 2007; Crowther et al. 2007). Population synthesis carried out by Lommen et al. (2005) suggests that $\sim 1 \mathrm{WR}+\mathrm{BH}$ binary, and $\sim 1 \mathrm{WR}+$ neutron star (NS) binary, may exist as bright X-ray sources in a galaxy like our own; this is certainly in keeping with the rarity of candidates.

Prestwich et al. (2007) re-analysed archival Gemini MultiObject Spectrograph spectra of the WR star associated with IC $10 \mathrm{X}-1$, and found evidence for a black hole $(\mathrm{BH})$ primary, with a plausible $\mathrm{BH}$ mass range of $23-34 M_{\odot}$. This result was subsequently confirmed by Silverman \& Filippenko (2008), who obtained spectra over five nights of observations with the Keck-I $10 \mathrm{~m}$ telescope. They report a BH mass of 20-35 $M_{\odot}$ for the compact object, making it the most massive known stellar $\mathrm{BH}$. NGC 300 X-1 has not yet been optically identified as a WR+BH binary; however, in this work we find striking similarities between the X-ray properties of NGC $300 \mathrm{X}-1$ and the confirmed $\mathrm{WR}+\mathrm{BH}$ binary IC $10 \mathrm{X}-1$. We will show that NGC $300 \mathrm{X}-1$ is likely to be a $\mathrm{WR}+\mathrm{BH}$ binary also.

$\mathrm{WR}+$ co binaries are just one subset of high mass X-ray binaries (HMXBs), and it is possible that their temporal and spectral characteristics closely resemble other types of HMXBs. Hence it is instructive to briefly review the pertinent properties of HMXBs in general. HMXBs are well classified by the nature 
of the donor star (see e.g. White et al. 1995, and references within).

Around $60 \%$ of Galactic HMXBs include a Be donor (Liu et al. 2007); these have elliptical orbits lasting tens to hundreds of days, only accreting near periastron. As a result, Be HMXBs are transient sources, generally powered by Bondi-Hoyle accretion (Bondi \& Hoyle 1944) from a decretion disc around the Be star (Okazaki \& Negueruela 2001). Several Bondi-Hoyle accreting HMXBs with a NS accretor exhibit rapid aperiodic variability in X-ray intensity with amplitude $\sim 10-30 \%$; their power density spectra (PDS) resemble a power law with spectral in$\operatorname{dex} \gamma \sim 1$ (power $\propto v^{-\gamma}$ where $v$ is the frequency, see e.g. Nagase 1989; Belloni \& Hasinger 1990; van der Klis 1995). These works suggested that such variability could be present in all accretionpowered pulsars. However, an inspection of the catalogues of HMXBs in the Galaxy and Magellanic clouds (Liu et al. 2005, 2007) revealed that only 16 out of 242 HMXBs have published records of aperiodic variability. HMXBs that have exhibited the variability described above have power law emission spectra with spectral index, $\Gamma, \sim 0.5-1.8$. There are no known $\mathrm{BH}$ HMXBs that accrete in this way.

A further $\sim 32 \%$ of Galactic HMXBs contain supergiant (SG) donors (Liu et al. 2007); the orbital periods range over 1.4-41 days, and the donor stars are (or are close to) filling their Roche lobe (see Kaper et al. 2004, for a review). The compact object in SG HMXBs is continuously accreting from the donor's wind and can be either wind-fed via Bondi-Hoyle accretion (with luminosities $\sim 10^{35}-10^{36} \mathrm{erg} \mathrm{s}^{-1}$ ) or disc-fed, reaching $\sim 10^{38} \mathrm{erg} \mathrm{s}^{-1}$ (Kaper et al. 2004).

Studies of Galactic disc-accreting XBs have shown that their spectral and variability properties depend as much on the accretion rate as the nature of the accretor (NS or BH). At low accretion rates, the emission spectra and temporal variability of discaccreting binaries are remarkably similar (van der Klis 1994). However, at certain, higher, accretion rates there are characteristic differences that may allow one to determine the nature of the primary; the PDS are well described by a power law with index $\gamma \sim 1$, but the emission spectra for NS and BH XBs are very different. For a disc-accreting XB with a NS primary, the emission corresponding to the power law PDS is non-thermal, while disc-accreting $\mathrm{BH}$ XBs exhibiting the same PDS have a thermal X-ray emission spectrum (van der Klis 1994). Indeed, Done \& Gierliński (2004) examined $\sim 1$ Tbyte of RXTE archival data on Galactic XBs, and found a region in colour-colour space that is uniquely associated with $\mathrm{BH} \mathrm{XBs}$ in this high, soft state. Hence, we may learn a great deal about disc-accreting binaries from their X-ray variability and emission spectra.

NGC $300 \mathrm{X}-1$ and IC $10 \mathrm{X}-1$ both have orbital periods $\sim 30 \mathrm{~h}$ and X-ray luminosities $\sim 10^{38} \mathrm{erg} \mathrm{s}^{-1}$ (Carpano et al. 2007; Prestwich et al. 2007; Silverman \& Filippenko 2008). We examined all existing XMM-Newton observations of NGC 300 $\mathrm{X}-1$ and IC $10 \mathrm{X}-1$, in order to compare their properties. In particular, we examined the PDS from the X-ray lightcurves of these systems for the first time. In Sect. 2 we describe the observations and data analysis, then provide the results from NGC 300 X-1 and IC 10 X-1 in turn in Sect. 3. We discuss our findings in Sect. 4, and consider four scenarios for NGC $300 \mathrm{X}-1$ and IC 10X-1: wind accretion onto a neutron star, disc accretion onto a neutron star, wind accretion onto a black hole and disc accretion onto a black hole. We then compare the properties of these sources with those of the ultraluminous X-ray sources (ULXs), many of which are thought to be HMXBs. Finally we draw our conclusions in Sect. 5.
Table 1. Journal of XMM-Newton observations of NGC $300 \mathrm{X}-1$ and IC $10 \mathrm{X}-1$. For each observation, we provide the target, date, revolution number, exposure and good time interval.

\begin{tabular}{ccccc}
\hline \hline Object & Date & Rev & Exp & GT \\
\hline NGC 300 X-1 & 2000 Dec. 26 & 0192 & $32 \mathrm{ks}$ & $25 \mathrm{ks}$ \\
NGC 300 X-1 & 2001 Jan. 01 & 0195 & $40 \mathrm{ks}$ & $40 \mathrm{ks}$ \\
NGC 300 X-1 & 2005 May 22 & 0998 & $35 \mathrm{ks}$ & 25 ks \\
NGC 300 X-1 & 2005 Nov. 25 & 1092 & $35 \mathrm{ks}$ & $35 \mathrm{ks}$ \\
IC 10 X-1 & 2003 Jul. 03 & 0653 & $44 \mathrm{ks}$ & $28 \mathrm{ks}$ \\
\hline
\end{tabular}

\section{Observations and data analysis}

Four XMM-Newton observations have been made of NGC $300 \mathrm{X}-1$, and one of IC $10 \mathrm{X}-1$; a journal of observations is provided in Table 1. For our analysis we used the XMM-Newton SAS version 7.0, and the FTOOLS suite, version 5.3.1. For each observation, we filtered out intervals of high background (flaring), using the criteria recommended by the SAS team. We note that no flaring occurred during the 2001, January or 2005, November observations of NGC 300 X-1.

We then extracted pn and MOS 0.3-10 keV lightcurves and spectra from a circular region centred on the source, along with corresponding response files. Background regions were then chosen, and lightcurves and spectra were obtained from these regions for pn and MOS also.

The pn and MOS source and background lightcurves were co-added, after careful synchronisation; XMM lightcurves are non-synchronised by default, leading to artificial variability if not treated properly (Barnard et al. 2007). Backgroundsubtracted lightcurves were analysed for variability, and PDS were made from the combined EPIC source lightcurves (background not subtracted). These lightcurves were averaged over several intervals of 1024 bins, with $5.2 \mathrm{~s}$ binning and geometric grouping; the sampled frequency range was $\sim 0.0002-0.1 \mathrm{~Hz}$.

The source and background emission spectra from the two MOS cameras were combined with the FTOOL mathpha to give MOS1+MOS2 source and background spectra, and the corresponding response matrices and ancillary response files were combined also, with addrmf and addarf. We modeled the pn and combined MOS spectra simultaneously using XSPEC 11.3, with a constant of normalisation to account for differences in the pn and MOS responses.

\section{Results}

We discuss our analysis of the variability and X-ray spectra from NGC $300 \mathrm{X}-1$ and IC $10 \mathrm{X}-1$ in the following sections.

\subsection{Variability of NGC $300 X-1$ and IC $10 X-1$}

The lightcurves from the four XMM-Newton observations of NGC 300 X-1 are well described by Carpano et al. (2007) and will not be discussed further here. Each observation reveals strong variability, with PDS that are well described by power laws with $\gamma \sim 1$. We present the 2005, November PDS in Fig. 1. The axes are log scaled, and normalised to give $\mathrm{rms}^{2}$ variability; the expected noise is subtracted. We note that no background flaring occurred in this observation, so there is no question of the PDS being an artefact of flaring or background filtering. This variability is certainly significant, as fitting the PDS with zero power yields a $\chi^{2}$ of 74 for 24 degrees of freedom (d.o.f.). We present the rms. variability and best fit $\gamma$ for each observation of 


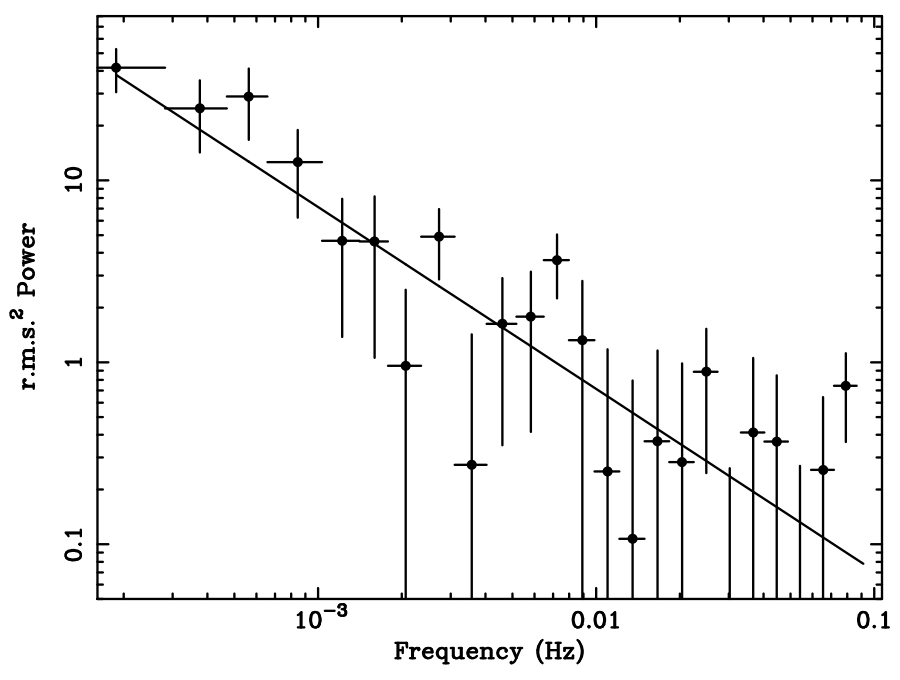

Fig. 1. PDS of the combined pn+MOS $0.3-10 \mathrm{keV}$ lightcurve of NGC 300 X-1 from the 2005 November XMM-Newton observation (Obs. 4). The axes are log scaled, and the y axis is normalised to give $\mathrm{rms}^{2}$ variability. The expected white noise level (7.48) is subtracted. A power law fit to the PDS is shown, with $\gamma=1$; the best fit $\chi^{2} /$ d.o.f $=$ $26 / 24$, with a good fit probability of 0.35 . Such a PDS is characteristic of a disc-accreting X-ray binary with a high accretion rate.

Table 2. Fits to the $0.3-10 \mathrm{keV}$ PDS of NGC $300 \mathrm{X}-1$ from Obs. 1-4. We give the observation number, as well the best fit spectral index with the $\chi^{2} /$ d.o.f.. We also give the $\chi^{2} /$ d.o.f. for zero power. Finally we give the fractional r.m.s. variability. We note that the PDS for Obs. 3 is more complex than for the other observations, due to an eclipse.

\begin{tabular}{ccccc}
\hline \hline Obs. & $\gamma$ & $\chi^{2} /$ d.o.f. & $\chi^{2} /$ d.o.f. (0) & Frac. rms $(\%)$ \\
\hline Obs. 1 & $1.30(12)$ & $17 / 23$ & $57 / 25$ & $50(3)$ \\
Obs. 2 & $1.3(2)$ & $27 / 23$ & $66 / 25$ & $44(2)$ \\
Obs. 3 & $1.8(2)$ & $56 / 23$ & $91 / 25$ & $38(2)$ \\
Obs. 4 & $1.05(11)$ & $23 / 23$ & $74 / 23$ & $22(2)$ \\
\hline
\end{tabular}

NGC $300 \mathrm{X}-1$ in Table 2. We note that the PDS from Obs. 3 is different to the PDS from the other observations; this difference is likely due to the eclipse that occurs in Obs. 3 (see Carpano et al. 2007).

We present the $0.3-10 \mathrm{keV}$, combined pn+MOS lightcurve of IC 10 X-1 in the top panel of Fig. 2; the background has been subtracted, and intervals of high background have been removed. The lightcurve has $100 \mathrm{~s}$ resolution. The system is highly variable throughout the observation, with a large intensity dip near the beginning. This dip may be intrinsic to the X-ray source, or due to an increase in line-of-sight absorption, such as an eclipse. The bottom panel shows the hardness ratio for each time bin, defined as the ratio of $2.5-10 \mathrm{keV}$ counts to $0.3-2.5 \mathrm{keV}$ counts. The hardness ratio is variable at the $3 \sigma$ level, and appears to be higher during the intensity dip at the start of the observation, hinting at photo-electric absorption; however, we cannot determine the phase of this intensity dip with the current orbital ephemeris. The variability is also consistent with stochastic variations in a source with a PDS described by $\gamma \sim 1$.

The PDS created from this lightcurve is presented in Fig. 3; as before, the $y$-axis is normalised to show the r.m.s. ${ }^{2}$ power, and the expected noise is subtracted. This PDS is also acceptably fitted by a power law with $\gamma=1$, with $\chi^{2} /$ d.o.f. $=35 / 24$. As with NGC $300 \mathrm{X}-1$, the r.m.s. variability is greater than for Galactic LMXBs, at $18 \pm 2 \%$, excluding the large intensity dip.

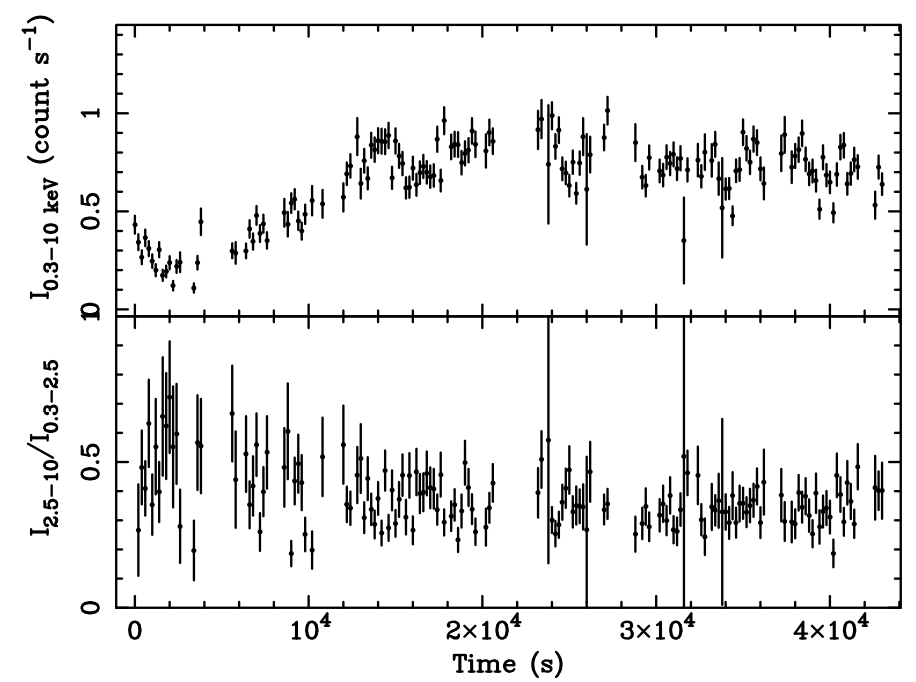

Fig. 2. Combined EPIC lightcurve of IC 10 X-1 from the 2003 XMMNewton observation (top); the binning is $100 \mathrm{~s}$. The lower panel shows the ratio of 2.5-10 keV photons to $0.3-2.5 \mathrm{keV}$ photons (hardness ratio) for each time bin. The lightcurve is clearly variable, and there is evidence for variability in the hardness ratio.

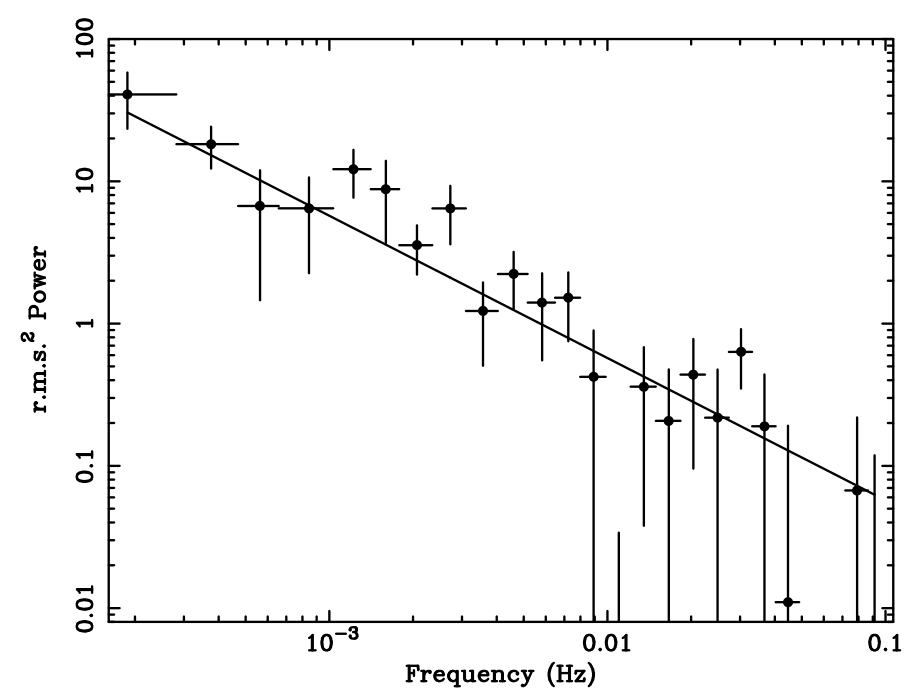

Fig. 3. Combined EPIC PDS of IC 10 X-1 from the 2003 XMM-Newton observation. The axes are log-scaled, and the $y$-axis is normalised to give $\mathrm{rms}^{2}$ variability. The expected noise level (3.49) is subtracted. The best fit power law with $\gamma=1$ is shown, for $\chi^{2} /$ d.o.f. $=35 / 24$.

The PDS observed from NGC $300 \mathrm{X}-1$ and IC $10 \mathrm{X}-1$ are characteristic of disc-fed $\mathrm{XBs}$ at high accretion rates, albeit with fractional r.m.s. variabilities $\sim 5-10$ times higher than observed in Galactic XBs (see e.g. van der Klis 1995). However, they are also similar to the variability observed in certain windfed HMXBs. Hence, we must examine the emission spectra of NGC 300 X-1 and IC 10 X-1 if we are to determine their natures.

\subsection{Modeling the spectra of NGC $300 X-1$ and IC $10 X-1$}

\subsubsection{NGC $300 \mathrm{X}-1$}

Carpano et al. (2007) modeled the spectra from the four observations of NGC $300 \mathrm{X}-1$ together. They used an absorbed, two-component model, consisting of a power law with photon index $\Gamma$, i.e. $N(E) \propto E^{-\Gamma}$, where $N$ is the number of photons 
Table 3. Fits to the spectra of NGC $300 \mathrm{X}-1$ from Obs. 1-4, and of IC $10 \mathrm{X}-1$. The first model is an absorbed power law plus Gaussian emission line, as favoured by Carpano et al. (2007a). For this model, we give absorption, normalised to $10^{22} \mathrm{H}$ atom $\mathrm{cm}^{-2}$, spectral index, line energy and width (in $\mathrm{keV}$ ), and $\chi^{2}$ /d.o.f., with good fit probability in square brackets. We only apply the Gaussian to NGC $300 \mathrm{X}-1$. We also give the $0.3-10 \mathrm{keV}$ luminosity normalised to $10^{37} \mathrm{erg} \mathrm{s}^{-1}$. We next provide the best fits for a disk blackbody + power law model that is often used when describing the high state of disc-accreting BH XBs. We give the absorption, temperature of the disk blackbody, spectral index of the power law component and $\chi^{2}$. We then give $0.3-10 \mathrm{keV}$ luminosity as before, and the fractional contribution of the power law model.

\begin{tabular}{|c|c|c|c|c|c|c|}
\hline Model & Parameter & Obs. 1 & $\begin{array}{l}\text { NGC } 300 \mathrm{X}-1 \\
\text { Obs. } 2\end{array}$ & Obs. 3 & Obs. 4 & IC $10 \mathrm{X}-1$ \\
\hline \multirow[t]{6}{*}{ PL + Line } & $n_{\mathrm{H}}$ & 0.036 & $0.067(12)$ & $0.052(9)$ & $0.07(2)$ & $0.75(3)$ \\
\hline & $\Gamma$ & $2.46(6)$ & $2.44(7)$ & 2.44 & $2.53(8)$ & $2.56(6)$ \\
\hline & $E_{\text {Line }}$ & 0.95 & $0.93(2)$ & $0.95(2)$ & $0.95(2)$ & $\ldots$ \\
\hline & $\sigma$ & 0.07 & $0.08(2)$ & 0.07 & $0.06(3)$ & \\
\hline & $\chi^{2} /$ d.o.f. & $100 / 60[9 \mathrm{E}-4]$ & $149 / 154$ [0.60] & $50 / 60[0.82]$ & $117 / 145[0.05]$ & $794 / 652$ [1E-4] \\
\hline & $L_{0.3-10 \mathrm{keV}}$ & $11.5(13)$ & $28.4(12)$ & $20(2)$ & $34.8(16)$ & $39(3)$ \\
\hline \multirow[t]{6}{*}{$\mathrm{BB}+\mathrm{PL}$} & $n_{\mathrm{H}}$ & $0.11(4)$ & $0.130(12)$ & $0.22(5)$ & $0.17(3)$ & $0.92(10)$ \\
\hline & $k T$ & $1.02(14)$ & $1.02(17)$ & $0.92(12)$ & $1.08(15)$ & $0.82(3)$ \\
\hline & $\Gamma$ & $3.41(6)$ & $3.01(16)$ & $3.8(3)$ & $3.26(6)$ & $3.9(5)$ \\
\hline & $\chi^{2} /$ d.o.f. & $52 / 58[0.67]$ & $211 / 155$ [2E-3] & $59 / 58[0.43]$ & $221 / 148[1.0 \mathrm{E}-4]$ & $697 / 650$ [0.09] \\
\hline & $L_{0.3-10 \mathrm{keV}}$ & $16.9(17)$ & $39(2)$ & $49(6)$ & $59(3)$ & $91(19)$ \\
\hline & Frac. PL & $0.80(11)$ & $0.93(14)$ & $0.91(18)$ & $0.92(6)$ & $0.93(3)$ \\
\hline \multirow[t]{6}{*}{$\mathrm{DISKBB}+\mathrm{PL}$} & $n_{\mathrm{H}}$ & $0.12(4)$ & $0.13(4)$ & $0.22(9)$ & $0.17(4)$ & $0.84(4)$ \\
\hline & $k T_{\text {in }}$ & $1.8(4)$ & $1.9(6)$ & $1.5(4)$ & $2.0(4)$ & 1.19(6) \\
\hline & $\Gamma$ & $3.6(7)$ & $3.1(4)$ & $4.1(7)$ & $3.4(3)$ & 3.9 \\
\hline & $\chi^{2} /$ d.o.f. & $56 / 58[0.54]$ & $213 / 155$ [1.3E-3] & $62 / 58[0.33]$ & $226 / 148[3.0 \mathrm{E}-5]$ & $710 / 651[0.05]$ \\
\hline & $L_{0.3-10 \mathrm{keV}}$ & $19(3)$ & $33(25)$ & $49(6)$ & $53(5)$ & $72(9)$ \\
\hline & Frac. PL & $0.89(17)$ & $0.6(7)$ & $0.91(18)$ & $0.93(11)$ & $0.86(19)$ \\
\hline
\end{tabular}

at energy $E$, and an emission line. They imposed identical values for $\Gamma$, line energy and line width for the four observations, with these values free to vary; the absorption and normalizations for each component were individually varied for each observation. For their best model, $\Gamma=2.44 \pm 0.03$, the line energy was $0.94 \pm 0.01 \mathrm{keV}$, with a width of $0.07 \pm 0.01 \mathrm{keV}$. They quote a reduced $\chi^{2}$ of 1.15 for 1033 d.o.f.; however, this equates to $\chi^{2} /$ d.o.f. $=1188 / 1033$, which is rejected at a $99.95 \%$ level and is therefore unacceptable. We therefore examined the spectra of NGC 300 X-1 more carefully.

For each observation of NGC $300 \mathrm{X}-1$, we modeled the pn and combined MOS spectra simultaneously, with each model consisting of a power law + emission line, suffering absorption and including a constant of normalization to account for differences in pn and MOS calibration. The resulting best fits are shown in Table 3. We found the spectra from Obs. 2-4 to be consistent with the model reported by Carpano et al. (2007). However, the best fit to the Obs. 1 spectra yielded $\chi^{2} /$ d.o.f. $=$ 100/60, an unacceptable fit. We show this best fit in Fig. 4, along with $\chi^{2}$ residuals. We see that the model systematically underestimates the emission at higher energies, suggestive of an additional emission component. Hence, we fitted all NGC 300 X-1 spectra with absorbed blackbody + power law models and also absorbed disc blackbody + power law models, commonly observed in NS and BH XBs respectively. The best fits for these models are also provided in Table 3; 0.3-10 keV luminosities are provided, assuming a distance of $1.88 \mathrm{Mpc}$ (Gieren et al. 2005). We see that both two-component models provided good fits to the Obs. 1 and Obs. 3 spectra. The Obs. 3 spectrum is well described by all three models; however, the resulting luminosities differ by factor of two $(\sim 5 \sigma)$; hence the nature of the Obs. 3 . emission spectrum is uncertain. Neither the Obs. 2 nor Obs. 4 spectra were successfully described by any thermal + power law

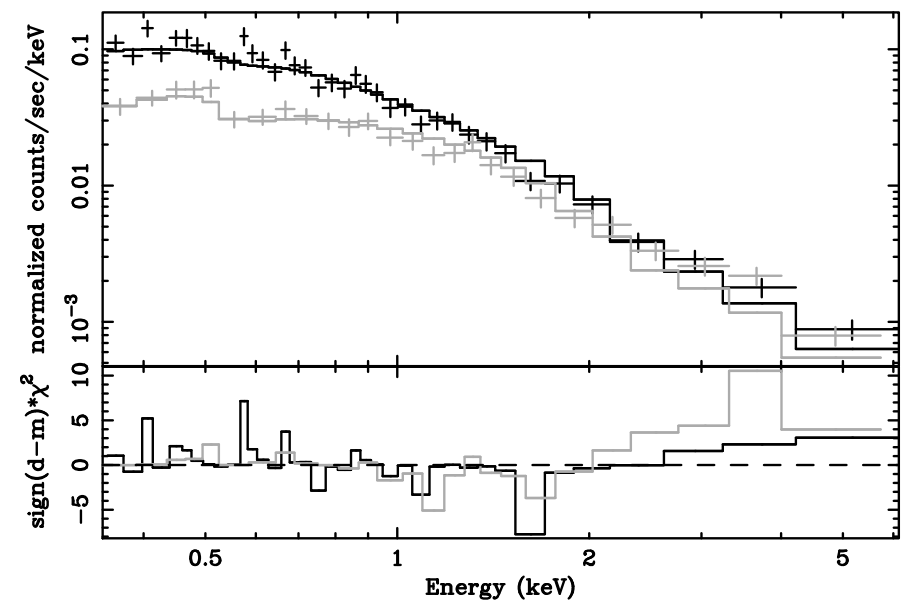

Fig. 4. Top panel: best fit model to the Obs. 1 pn (black) and combined MOS (grey) $0.3-10 \mathrm{keV}$ spectra of NGC 300 X-1 using a power law with $\Gamma=2.44$ and a $0.95 \mathrm{keV}$ Gaussian line with width $0.07 \mathrm{keV}$; $\chi^{2} /$ d.o.f. $=100 / 60$, which is unacceptable. Bottom panel: $\chi^{2}$ residuals, showing a hard excess; this shows that a flatter power law and/or a second emission component is required. The spectrum of NGC 300 $\mathrm{X}-1$ from Obs. 1 is certainly different from the model obtained by Carpano et al. (2007) when fitting spectra from all four observations simultaneously.

model; hence NGC $3000 \mathrm{X}-1$ exhibits at least two spectral states: one exhibited in Obs. 1 and the other exhibited in Obs. 2 and 4.

Finally, we fitted the spectra with the hard power law emission that is characteristic of Bondi-Hoyle accretion onto a neutron star. Our literature review shows that the wind-accreting HMXBs with PDS similar to those observed from NGC 300 X-1 all have spectra that are well described by a power law with $\Gamma \leq 1.8$. Hence, we modeled the spectra from each observation 


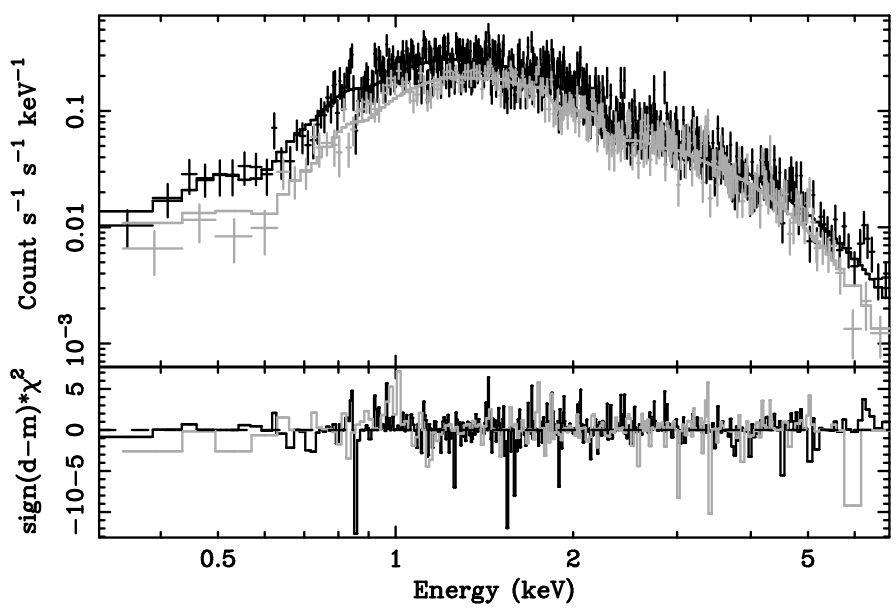

Fig. 5. Simultaneous fits to the pn (black) and combined MOS (grey) spectra of IC 10 X-1 from the 2003 XMM-Newton observation with the best fit emission model; the first $20 \mathrm{ks}$ of the observation was excluded. The top panel shows the spectra modeled with a power law with photon index $3.5 \pm 0.2$ and a $0.86 \pm 0.03 \mathrm{keV} \mathrm{keV}$ blackbody, suffering absorption equivalent to $8.6 \pm 0.4 \times 10^{21} \mathrm{H}$ atom $\mathrm{cm}^{-2}$, with $\chi^{2}=849$ for 840 degrees of freedom (good fit probability 0.4 ). The bottom panel shows the $\chi^{2}$ residuals.

with power law models, restricting $\Gamma$ to be $<1.8$. We found the best fit $\chi^{2} /$ d.o.f. to be $\gtrsim 5$ in each case. Hence NGC $300 \mathrm{X}-1$ is unlikely to be powered by Bondi-Hoyle accretion onto a neutron star.

\subsubsection{IC $10 \mathrm{X}-1$}

We first attempted to simultaneously fit the pn and combined MOS spectra of IC 10 X-1 with various emission models, but failed to obtain a good fit. This is likely to be due to the fact that IC $10 \mathrm{X}-1$ varies in colour and intensity during the observation, mixing the spectral states so that a physical fit is impossible.

In light of this, we excluded the first $20 \mathrm{ks}$ of the observation, where the intensity and colour of IC $10 \mathrm{X}-1$ is unstable. We modeled the spectrum of the remaining $\sim 22 \mathrm{ks}$ with power law, blackbody + power law, and disc blackbody + power law emission models, all suffering absorption. The best fits are presented in Table 3; quoted luminosities assume a distance of $740 \mathrm{kpc}$ (Demers et al. 2004). The disc blackbody + power law model favoured an unreasonably high $\Gamma$, although $\Gamma$ was not well constrained. Hence, we fixed it to 3.9 , the best fit $\Gamma$ for the blackbody + power law model. No simple power law model was able to fit, but both two-component models provided acceptable fits. However, since we know that IC 10 X-1 contains a BH, we favour the disc blackbody + power law model.

Wang et al. (2005) modeled pn and MOS spectra from the entire XMM-Newton observation, mixing spectral states and thereby producing a spectrum that is not representative of either state. As a result, they required a metal abundance $<0.01$ Solar to achieve good fits; they remark that such a low metallicity is probably not physical, but attribute it to poor calibration at low energies rather than to spectral mixing. Their preferred model is a self-consistent Comptonised multi-colour disc blackbody where an accretion disc emits a multi-temperature disc blackbody, which is Comptonised by a spherically symmetric corona of hot electrons around the disc. Hence Wang et al. (2005) also favour a non-thermal emission model for IC 10 X-1. They estimated the mass of the black hole by assuming that the inner disc radius corresponds to the innermost stable orbit, after correcting for relativistic effects. They obtained a $\mathrm{BH}$ mass range of $\sim 4 M_{\odot}$ for a stationary $\mathrm{BH}$, up to $\sim 30 M_{\odot}$ for a $\mathrm{BH}$ with extreme spin; this range overlaps the mass range found by Silverman \& Filippenko (2008).

\section{Discussion}

We have examined the variability and emission spectra of one $\mathrm{BH}+\mathrm{WR}$ candidate (NGC $300 \mathrm{X}-1$ ), and the newly confirmed $\mathrm{BH}+\mathrm{WR}$ system IC $10 \mathrm{X}-1$. The $0.3-10 \mathrm{keV}$ spectrum of Obs. 1 of NGC $300 \mathrm{X}-1$ is strikingly similar to that of IC $10 \mathrm{X}-1$. This lends strong support to the case for NGC $300 \mathrm{X}-1$ being a BH+WR system. Both systems exhibit variability that scales with frequency, $v$, as $v^{-1}$, identified with $\mathrm{BH} \mathrm{XBs}$ in the high state. However, their $0.3-10 \mathrm{keV}$ emission spectra appear to be $\sim 90 \%$ non-thermal, while the canonical high state BH spectrum is thermally dominated. We therefore suggest that these systems exist in a previously unrecognised state.

\subsection{Accretion scenarios}

We next discuss different accretion scenarios. IC $10 \mathrm{X}-1$ has been confirmed as a WR+BH binary, hence we first discuss wind and disc accretion onto a BH. NGC 300 X-1 has an unknown accretor, and may contain a neutron star; therefore, we also discuss wind and disc accretion onto a NS.

Since the emission from Bondi-Hoyle accreting neutron stars appears to originate on or near the surface of the neutron star, it is unclear what X-ray emission could be expected from BondiHoyle accretion onto a black hole. Indeed, there are no known black hole Be XBs, and all known black hole supergiant XBs exhibit accretion discs. Furthermore, the modeling of BondiHoyle accretion onto black holes requires relativistic, threedimensional magnetohydrodynamical modeling (see. e.g. Font et al. 1999, and references within). These models have not yet produced unambiguous predictions for the variability or emission mechanisms expected from such systems. Hence, there are no observational or theoretical constraints on the spectral shape or variability exhibited by HMXBs powered by Bondi-Hoyle accretion onto a $\mathrm{BH}$.

The short orbital periods $(\sim 30 \mathrm{~h})$ and high X-ray luminosities of NGC 300 X-1 and IC 10 X-1 resemble those of discaccreting SG HMXBs (Kaper et al. 2004); we therefore consider them likely disc-accretors also. Prestwich et al. (2007) calculated the range of orbital periods that permit Roche lobe overflow for IC $10 \mathrm{X}-1$. They obtained periods of $\sim 2.5-3 \mathrm{~h}$, and rejected disc accretion for IC $10 \mathrm{X}-1$ (this also applies to NGC $300 \mathrm{X}-1$, as it has a similar orbital period to IC $10 \mathrm{X}-1)$. However, it is entirely possible for the winds of the Wolf-Rayet stars to power disc accretion. The three known black hole HMXBs Cygnus X-1, LMC X-1 and LMC X-3 are all disc-accreting, with orbital periods of $5.60 \mathrm{~d}, 4.22 \mathrm{~d}$ and $1.70 \mathrm{~d}$ respectively (see e.g. Lewin et al. 1995, and references within). Of these, only LMC X-3 is thought to be Roche lobe filling. NGC 300 X-1 and IC 10 X-1 have shorter orbital periods than any of these systems, so disc accretion onto a $\mathrm{BH}$ in these systems is entirely plausible.

As discussed in Sect. 3, our spectral modeling of NGC 300 $\mathrm{X}-1$ allows us to rule out Bondi-Hoyle (wind) accretion onto a $\mathrm{NS}$, as power law spectral fits with $\Gamma \leq 1.8$ yield $\chi^{2} /$ d.o.f. $>$ 5 for all observations. However, the emission and variability of NGC 300 X-1 are in keeping with a disc-fed NS XB. The donor star may be the WR star, in which case, the disc would likely 
be wind-fed. Alternatively, NGC $300 \mathrm{X}-1$ could be a bright NS LMXB which is merely coincident with the WR.

\subsection{A new black hole state?}

We prefer disc accretion scenarios for IC $10 \mathrm{X}-1$ and NGC 300 $\mathrm{X}-1$, although there is no independent evidence for a disc, such as radio jets. All known $\mathrm{BH} \mathrm{XBs}$ are disc accreting, yet the properties exhibited by IC $10 \mathrm{X}-1$ and NGC $300 \mathrm{X}-1$ are as yet unrecognised. Their observed emission spectra most closely resemble the very high (steep power law, SPL) state (van der Klis 1995; McClintock \& Remillard 2006). However, the PDS of sources in the SPL state are characterised by broken power laws, where $\gamma$ changes from $\sim 0$ at low frequencies to $\sim 1$ at high frequencies; often quasi-periodic oscillations (QPOs) are observed (McClintock \& Remillard 2006).

We note that Cyg X-3, the other WR+co candidate, exhibits similar timing behaviour to NGC $300 \mathrm{X}-1$ and IC $10 \mathrm{X}-1$. Some believe that scattering in the wind suppresses high frequency variability (see e.g. Kitamoto et al. 1992), possibly masking a SPL PDS. Hence, one might wonder if NGC 300 X-1 (in Observation 1) or IC 10 X-1 are disguising SPL states. However, the r.m.s. variability of NGC 300 X-1 was already $44 \%$ in Observation 1, while the r.m.s. variability of IC 10 X-1 was $18 \%$. Hence they are already considerably more variable than BH LMXBs in the very high state (van der Klis 1995), making this hypothesis unlikely.

Instead, we believe that the observed behaviour maybe due to the status of the corona. In NS LMXBs, the self-irradiation of the disc is more efficient than in BH LMXBs, hence NS LMXBs have hot, stable discs, while all BH LMXBs are transient (King et al. 1997); they spend the majority of their time in a quiescent state, with outbursts generally lasting a few months (see e.g. McClintock \& Remillard 2006). During the rise of the outburst, a BH LMXB will go from a low accretion rate, hard state with associated radio jets, to a high accretion rate, soft state with no jets (see e.g. Homan \& Belloni 2005, and references within). It is therefore possible that the source of the hard, non-thermal emission, and also of the jets in BH LMXBs (e.g. the corona) is ejected during this transition; indeed Gallo et al. (2004) witness a large radio flare that coincided with a hard to soft transition in GX 339-4.

By contrast, the three known Galactic BH HMXBs are all persistently bright. The HMXB accretion discs are expected to be small, and hence will be more easily kept hot at the outer edge, making them stable.

Therefore, the behaviour of IC $10 \mathrm{X}-1$ and NGC $300 \mathrm{X}-1$ may be explained by stable disc accretion. A small fraction of the $\sim 10^{-5}-10^{-4} M_{\odot} \mathrm{yr}^{-1}$ wind from a WR companion leaving the vicinity of the L1 point could provide enough mass, with sufficient angular momentum, to sustain an accretion disc that is persistently in the high state. In this case, the corona could be retained, and the emission would then be dominated by a nonthermal component due to inverse-Comptonisation of cool photons on hot electrons, as seen in NS XBs at high accretion rate.

Observations of the Galactic BH HMXBs support this hypothesis. LMC X-1 has never been observed in the low/hard state (see e.g. Wilms et al. 2001; Yao et al. 2005). Nowak et al. (2001) report "high state" (i.e. power $\propto v^{-1}$ ) PDS from RXTE observations of LMC X-1, while Wilms et al. (2001) find its emission spectrum to be dominated by a power law with photon index $\gtrsim 3$, with a $0.8-1.1 \mathrm{keV}$ blackbody component. This emission spectrum is remarkably similar to our best fit models for NGC 300 X-1 in Obs. 1 , and to IC 10 X-1. In contrast, Cyg X-1 and LMC
X-3 exhibit transitions between the low/hard and high states, and exhibit canonical spectra (McClintock \& Remillard 2006, and references within).

\subsection{Comparison with ULXs}

It is interesting to compare these systems with the ULXs, as many ULXs are thought to be HMXBs. In their review of black hole masses and spectral states in ULXs, Soria \& Kuncic (2008) find that only $\sim 10 \%$ of sources with luminosities above $10^{39} \mathrm{erg} \mathrm{s}^{-1}$ exhibit high/soft (i.e. thermally dominated) spectra, with almost all ULXs instead dominated by a broad "power law-like" component that contributes $\sim 90 \%$ of the flux. Such characteristics are remarkably similar to those of NGC $300 \mathrm{X}-1$ and IC $10 \mathrm{X}-1$, where the power law contributes $\sim 80-90 \%$ of the flux. It is therefore possible that these ULXs could also be peresistently bright with stable coronae. It is therefore instructive to study the spectral and, crucially, timing properties of ULXs.

Winter et al. (2006) conducted a survey of all point sources $>10^{38} \mathrm{erg} \mathrm{s}^{-1}$ in XMM-Newton observations of 32 nearby galaxies. They modeled the spectra of $\sim 100 \mathrm{X}$-ray sources, each with $\gtrsim 400$ counts. Ten sources exhibited spectra consistent with NGC 300 X-1 in Obs. 2 and 4, including three ULXs. A further 23 sources exhibited spectra consistent with Obs. 1 of NGC 300 X-1 and IC 10 X-1, including 10 ULXs. Hence the processes in NGC $300 \mathrm{X}-1$ and IC $10 \mathrm{X}-1$ could also occur in some ULXs.

While most of the known ULXs are too faint to allow fruitful analysis of the PDS, several of the nearest ULXs are as bright as, or brighter than, NGC $300 \mathrm{X}-1$ and IC $10 \mathrm{X}-1$. A full analysis of the timing properties of nearby ULXs is beyond the scope of this work, and is the subject of a forthcoming paper. However, we conducted a brief literature review of ULX variability. Several ULXs exhibit QPOs, e.g. NGC 5408 X-1 (Strohmayer et al. 2007), M82 X-1 (Strohmayer \& Mushotzky 2003) and HoIX X-1 (Dewangan et al. 2006). Strohmayer et al. (2007) found the NGC 5408 X-1 PDS to be characterised by a broken power law; this PDS, along with the emission spectrum (dominated by a power law with $\Gamma \sim 2.6$ ) is reminiscent of the SPL state of Galactic black holes, unlike NGC 300 X-1 and IC 10 X-1. The published PDS of M82 X-1 and HoIX X-1 do not extend to sufficiently low frequencies to determine whether they are also in the SPL state. We note that the broken power law PDS and power law PDS identified by Cropper et al. (2004) in NGC 4559 X-7 and NGC4559 X-10 are both artificial, caused by improper treatment of non-sychronised lightcurves (Barnard et al. 2007).

\section{Conclusions}

IC $10 \mathrm{X}-1$ is the first confirmed BH+WR system, and NGC 300 $\mathrm{X}-1$ is thought to be one also. They are known to have strikingly similar X-ray periods and luminosities, so we studied them in detail. We have examined for the first time the PDS of NGC 300 $\mathrm{X}-1$, in four XMM-Newton observations, and also of IC $10 \mathrm{X}-1$, in one XMM-Newton observation. We find that they are well described by a simple power law with spectral index $\simeq 1$, over the $0.002-0.1 \mathrm{~Hz}$ range; such variability is characteristic of discaccreting XBs in their high state, or of some HMXB pulsars that are powered by Bondi-Hoyle accretion. We also find a striking resemblance between the Obs. 1 NGC $300 \mathrm{X}-1$ spectrum and the IC $10 \mathrm{X}-1$ spectrum. Since IC $10 \mathrm{X}-1$ is a confirmed WR+BH system, our results provide strong support for NGC $300 \mathrm{X}-1$ being one too. 
We have considered four accretion scenarios: disc accretion or Bondi-Hoyle accretion, onto a BH or NS. We favour disc accretion onto a $\mathrm{BH}$ for both systems, but cannot rule out Bond-Hoyle accretion onto a BH. The observed X-ray spectra of NGC 300 X-1 allowed us to rule out Bondi-Hoyle accretion onto a NS, but do not exclude disc accretion onto a NS+WR binary, or onto a NS LMXB that is merely coincident with the WR.

If both NGC $300 \mathrm{X}-1$ and IC $10 \mathrm{X}-1$ are indeed WR+BH binaries, then they are spectrally distinct from the known BH XBs. We propose that this difference may be due to these systems being persistently in the high state, allowing them to keep their disc corona, like LMC X-1; contrariwise, BH LMXBs enter the high state only in violent outbursts, where the corona may be ejected. NGC 300 X-1 and IC 10 X-1 (and possibly LMC X-1) would then comprise a new class of $\mathrm{BH}$ binary, defined by their high mass transfer rates and consequent stable accretion disc configurations. This stable disc scenario may also explain why only $\sim 10 \%$ of ULXs exist in the thermally-dominated high soft state. A fair comparison between the ULXs and NGC $300 \mathrm{X}-1$ and IC $10 \mathrm{X}-1$ requires detailed analyis of the variability of ULXs in addition to spectral analysis; we will carry this out in a future paper.

Acknowledgements. PDS were fitted using fitpowspec, provided by P. J. Humphrey. Astrophysics at the Open University is funded by a STFC (formerly PPARC) rolling grant. We thank Paul Crowther and Andrea Prestwich for communicating results prior to publication. We are also grateful to the anonymous referee for a very constructive review of the paper.

\section{References}

Barnard, R., Trudolyubov, S., Kolb, U. C., et al. 2007, A\&A, 469, 875

Bauer, F. E., \& Brandt, W. N. 2004, ApJ, 601, L67

Belloni, T., \& Hasinger, G. 1990, A\&A, 227, L33

Bondi, H., \& Hoyle, F. 1944, MNRAS, 104, 273

Carpano, S., Pollock, A. M. T., Wilms, J., Ehle, M., \& Schirmer, M. 2007, A\&A, 461, L9
Clark, J. S., \& Crowther, P. A. 2004, A\&A, 414, L45

Cropper, M., Soria, R., Mushotzky, R. F., et al. 2004, MNRAS, 349, 39 Crowther, P. A. 2007, ARA\&A, 45, 177

Crowther, P. A., Carpano, S., Hadfield, L. J., \& Pollock, A. M. T. 2007, A\&A, 469, L31

Demers, S., Battinelli, P., \& Letarte, B. 2004, A\&A, 424, 125

Dewangan, G. C., Griffiths, R. E., \& Rao, A. R. 2006, ApJ, 641, L125

Done, C., \& Gierliński, M. 2004, Progress of Theoretical Physics Supplement, 155,9

Font, J. A., Ibáñez, J. M., \& Papadopoulos, P. 1999, MNRAS, 305, 920

Gallo, E., Corbel, S., Fender, R. P., Maccarone, T. J., \& Tzioumis, A. K. 2004, MNRAS, 347, L52

Gieren, W., Pietrzyński, G., Soszyński, I., et al. 2005, ApJ, 628, 695

Homan, J., \& Belloni, T. 2005, Ap\&SS, 300, 107

Kaper, L., van der Meer, A., \& Tijani, A. H. 2004, in Rev. Mex. Astron. Astrofis. Conf. Ser. 21, ed. C. Allen \& C. Scarfe, 128

King, A. R., Kolb, U., \& Szuszkiewicz, E. 1997, ApJ, 488, 89

Kitamoto, S., Mizobuchi, S., Yamashita, K., \& Nakamura, H. 1992, ApJ, 384, 263

Lewin, W. H. G., van Paradijs, J., \& van den Heuvel, E. P. J. 1995, X-ray Binaries (Cambridge University Press)

Liu, Q. Z., van Paradijs, J., \& van den Heuvel, E. P. J. 2005, A\&A, 442, 1135

Liu, Q. Z., van Paradijs, J., \& van den Heuvel, E. P. J. 2007, A\&A, 469, 807

Lommen, D., Yungelson, L., van den Heuvel, E., Nelemans, G., \& Portegies Zwart, S. 2005, A\&A, 443, 231

McClintock, J. E., \& Remillard, R. A. 2006, Black hole binaries, Compact stellar X-ray sources, 157

Nagase, F. 1989, PASJ, 41, 1

Nowak, M. A., Wilms, J., Heindl, W. A., et al. 2001, MNRAS, 320, 316

Okazaki, A. T., \& Negueruela, I. 2001, A\&A, 377, 161

Prestwich, A. H., Kilgard, R., Crowther, P. A., et al. 2007, ApJ, 669, L21

Silverman, J. M., \& Filippenko, A. V. 2008, ApJ, 678, L17

Soria, R., \& Kuncic, Z. 2008, Advances in Space Research, 42, 517

Strohmayer, T. E., \& Mushotzky, R. F. 2003, ApJ, 586, L61

Strohmayer, T. E., Mushotzky, R. F., Winter, L., et al. 2007, ApJ, 660, 580

van der Klis, M. 1994, ApJS, 92, 511

van der Klis, M. 1995, X-ray Binaries (Cambridge University Press), 256

Wang, Q. D., Whitaker, K. E., \& Williams, R. 2005, MNRAS, 362, 1065

White, N. E., Nagase, F., \& Parmar, A. N. 1995, X-ray Binaries (Cambridge University Press)

Wilms, J., Nowak, M. A., Pottschmidt, K., et al. 2001, MNRAS, 320, 327

Winter, L. M., Mushotzky, R. F., \& Reynolds, C. S. 2006, ApJ, 649, 730

Yao, Y., Wang, Q. D., \& Nan Zhang, S. 2005, MNRAS, 362, 229 